\title{
Article \\ Topological Sigma-Semiring Separation and Ordered Measures in Noetherian Hyperconvexes
}

Susmit Bagchi

check for updates

Citation: Bagchi, S. Topological Sigma-Semiring Separation and Ordered Measures in Noetherian Hyperconvexes. Symmetry 2022, 14, 422. https://doi.org/10.3390/ sym14020422

Academic Editor: Basil

Papadopoulos

Received: 31 January 2022

Accepted: 18 February 2022

Published: 20 February 2022

Publisher's Note: MDPI stays neutral with regard to jurisdictional claims in published maps and institutional affiliations.

Copyright: (C) 2022 by the author. Licensee MDPI, Basel, Switzerland. This article is an open access article distributed under the terms and conditions of the Creative Commons Attribution (CC BY) license (https:// creativecommons.org/licenses/by/ $4.0 /)$.
Department of Aerospace and Software Engineering (Informatics), Gyeongsang National University, Jinju 660-701, Korea; profsbagchi@gmail.com

\begin{abstract}
The interplay between topological hyperconvex spaces and sigma-finite measures in such spaces gives rise to a set of analytical observations. This paper introduces the Noetherian class of $k$ finite $k$-hyperconvex topological subspaces (NHCs) admitting countable finite covers. A sigma-finite measure is constructed in a sigma-semiring in a NHC under a topological ordering of NHCs. The topological ordering relation maintains the irreflexive and anti-symmetric algebraic properties while retaining the homeomorphism of NHCs. The monotonic measure sequence in a NHC determines the convexity and compactness of topological subspaces. Interestingly, the topological ordering in NHCs in two isomorphic topological spaces induces the corresponding ordering of measures in sigma-semirings. Moreover, the uniform topological measure spaces of NHCs need not always preserve the pushforward measures, and a NHC semiring is functionally separable by a set of inner-measurable functions.
\end{abstract}

Keywords: topological spaces; sigma-semiring; measure spaces; convex; Noetherian class

MSC: 54F05; 54E15; 28C15

\section{Introduction}

The interplay between topological spaces, Borel sets, Baire categorization and measurability in a $\sigma$-semiring structure is interesting as well as complex. The interactions between topology and measure theory are generally formulated by forming the smallest $\sigma-$ field, where the compactness of a subspace facilitates the computation of measure [1] It is known that a metrizable space may be separable or may not be separable, affecting the measures in the $\sigma$-semiring structures within the spaces. This effectively gives rise to the formation of a Borel hierarchy in metrizable spaces [2]. Note that the Borel sets as well as Baire sets are members of $\sigma$-algebra generated by a set of subspaces in a topological space $X$. The existence of scattering in a topological space affects Borel classification as well as measures. A topological space $X$ is called a scattered space if $\forall A \subset X, \exists a \in A$ such that $A \cap(U \subset X)=\{a\}$ where $U=U^{o}$ (here, $U^{o}$ represents the interior of a respective open set). If we consider a continuous function $f: X \rightarrow Y$ from a topological space $X$ to a Hausdorff topological space $Y$, then an interesting question arises: what is the condition to form a function $f($.$) in Baire first class? The answer is mainly two-fold in view of topology$ and convexity: (1) if $X$ is a metric space and $Y$ is a convex subspace of a Banach space, and (2) if $X$ is a normal topological space and $Y=R$, where $R$ is a set of real numbers. Interestingly, the connectedness of a topological space has a role in this case. For example, the properties of Baire first category are preserved by $f: X \rightarrow Y$ if the topological space $X$ is normal and the topological space $Y$ is arc-connected [2]. If a space is metrizable, then one can find $\sigma$-discrete bases within the space affecting the measurability. A function $f: X \rightarrow Y$ induces the co- $\sigma$-discrete bases in $Y$, given as $\left\{f\left(U^{o}\right): U^{o} \subset X\right\}$, if $X$ has respective $\sigma$-discrete bases [3]. Note that the concept of $\sigma$-discrete bases in a space can be extended to the concept of hyper-Borel sets in a space [3]. Interestingly, the continuous 
Borel measurable functions between non-separable spaces do not necessarily preserve the structures of $\sigma$-discrete bases. Similarly, the interplay between the topological homeomorphism and Borel isomorphism shows that not all topological properties are retained in Borel isomorphic spaces. A bijection $f: X \rightarrow Y$ is called Borel isomorphic if $f, f^{-1}$ are both Borel. Moreover, every Borel measurable function is analytically measurable (i.e., Souslin measurable) [4]. However, it is found that every topological homeomorphism is a Borel isomorphism, but the converse is not always true [4]. As a consequence, one cannot guarantee that the measurability can be preserved in a generalized topological space, even if the Borel isomorphism is attained by $f: X \rightarrow Y$. Moreover, the Borel measure need not be always real valued in a topological space. For example, the complex-valued Borel measures exist in a Minkowski topological space, which allows computing densities with respect to the Lebesgue measure [5].

\section{Motivation and Contributions}

The developments in topological measure theory are propelled by Alexandrov and Varadarajan, considering that the topological spaces are always completely regular as well as Hausdorff $[6,7]$. The fundamental question in measure theory and its topological variants is the extensibility of $\sigma$-algebras [8]. The approach of Alexandrov is based upon the finitely additive set-valued functions in a topological space, and the approach of Varadarajan is primarily based upon the $C^{b}$ algebraic forms of bounded continuous real-valued functions in the completely regular spaces. Kirk and Crenshaw further generalized the $C^{b}$ algebraic approach by introducing the concept of paving $W(X)$ in a space $X$ and then constructing a $\sigma$-ring based on the paving [6]. However, the concept of paving has a strong flavor of general topology, and the corresponding topological measure is finitely $W$ - regular. Moreover, the structure of $\sigma$-ring depending on $W(X)$ is a modification of a standard $\sigma$-semiring in a topological space. Furthermore, the algebra-based topological separation of subspaces also depends on $W(X)$. In the case of a completely regular topological space, an extremely disconnected space (i.e., closure of open set is open) exists, where the corresponding Baire sets become reduced and the zero-sets are easy to identify [7]. In other words, the topological determination of measure compactness becomes simpler in this setting. It is shown that topological measures and deficient measures may not always support subadditivity and the properties of linear functionals while admitting the weak convergence of topological measures, which is a variety of Alexandrov weak convergence [9]. Interestingly, if we consider a ring of sets $\sigma(A)$ and a topological vector space $X$, then the measure $\mu: \sigma(A) \rightarrow X$ may show strong convergence to zero if $\mu\left(\langle B\rangle_{i=1}^{n}\right) \rightarrow 0$ in $\sigma(A)$ where the sets in sequence $\langle B\rangle_{i=1}^{n}$ under measure are disjoint [10]. These observations are the motivation to investigate the properties of topological measure in the topologically ordered spaces under an anti-symmetric ordering relation. Moreover, it is interesting to analyze the inherent topological properties, such as invariances and measure sequences, if the topological spaces are hyperconvex Noetherian varieties. The interesting questions are as follows: (1) How do we formulate an irreflexive and anti-symmetric topological ordering relation between two Noetherian classes? (2) What are the properties of topological measures in such Noetherian hyperconvex classes under topological ordering relation? (3) What are the properties of a topological measure sequence in the hyperconvex space? This paper addresses these questions and presents the analytical results by combining the elements of topology and measure theory.

The main contributions made in this paper can be summarized as follows. A Noetherian $k$-hyperconvex class (NHC) in a Hausdorff topological space is constructed such that every local neighborhood basis is countably coverable, and a fiber can be suitably attached for finite $k$. A topological ordering relation is introduced between two NHCs, where the ordering relation is irreflexive, anti-symmetric and transitive without affecting the homeomorphism of topological spaces. This paper proposes a set of analytical properties of finite measures in sigma-semirings under the topological ordering relation in NHCs. We 
show that pushforward measures are not always maintainable, and the sigma-semiring is topologically separable by inner-measurable functions.

The rest of the paper is organized as follows. The preliminary concepts and a set of existing classical results are presented in Section 2. The proposed definitions of topological structures are presented in Section 3. The analytical results are presented in Section 4. Finally, Section 5 concludes the paper.

\section{Preliminaries}

In general, a real-valued measure is formulated based on the algebraic semiring structure on sets. The generalized algebraic structure of the semiring on a set $S$ is given by $\langle S,+, \cdot\rangle$ where $\langle S,+\rangle$ is a commutative semigroup, $\langle S, \cdot\rangle$ is a semigroup and the multiplication $\cdot: S^{2} \rightarrow S$ distributes over $+: S^{2} \rightarrow S$ within the structure. The concept of the zero-set plays important roles in the inter-relationship between the algebraic semiring structure and the topological space, which is defined as follows [11].

Definition 1 (zero-set in topological space). Let $X$ be a Hausdorff topological space and $f: X \rightarrow R$ be a real-valued function. The zero-set in the topological space $A \subset X$ is defined to be a subset such that $A=f^{-1}(0)$.

The co-zero set is the complement of the zero-set, which is denoted as $\operatorname{coz}(A)$. The set of continuous functions in a topological space can generate a $\sigma$-semiring structure. As a result, we can define the zero-set in a topological space alternatively as presented the following definition $[2,12]$.

Definition 2 (semiring zero-set in topological space). If $X$ is a topological space and $C(X,(0, \infty])$ denotes a set of continuous functions generating a semiring in the topological space $X$, then a closed set $C l(f)$ of a function $f($.$) is the zero-set such that C l(f)=f^{-1}(0)$.

It is well known that a Hausdorff topological space $X$ is a Tychonoff space if every subspace $B \subseteq X$ and a point $a \in X \backslash B$ are functionally separable, where $B=\bar{B} \neq \phi$. Note that $\bar{B}$ is the closure of the corresponding set. Suppose we consider a family of subspaces $F$ in a Tychonoff topological space $X$. Hence, we can define the concept of measurability, which is given as follows [2].

Definition 3 (measurable topological subspaces). Let $X$ be a set and $Y$ be a Tychonoff space. A function $f: X \rightarrow Y$ is called $F$-measurable if $[U \subset Y] \Rightarrow\left[f^{-1}(U) \in F\right]$ where $U=U^{o}$.

It is important to note that not all subspaces are measurable. For example, the Bernstein set, which is a Baire-Lindelöf variety, is not measurable [11]. In a linear space, the convexity of functionals and bounded real-valued linear functions have an interesting relationship in terms of measures. Suppose $\omega: X \rightarrow R \cup\{+\infty\}$ is an increasing functional on the linear space of real-valued functions with convexity [13]. If we consider two functions $f$ and $g$ in the space $X$, then the convexity of $\omega: X \rightarrow R \cup\{+\infty\}$ satisfies the condition given by $[f \geq g] \Rightarrow[\omega(f) \geq \omega(g)]$. Let us consider that $X$ is a family of continuous real-valued functions on a topological space $A$ represented as $f: A \rightarrow R$. If the measure $\mu: \sigma(X) \rightarrow R$ is finite, then it results in the following theorem [13].

Theorem 1. Every finite measure $\mu: \sigma(X) \rightarrow R$ is regular and closed. Moreover, if $\left\langle B_{n}\right\rangle_{n=1}^{m}$ is a sequence of compact sets in $\sigma(X)$ such that the measure preserves $\mu\left(\left\langle B_{n}\right\rangle_{n=1}^{m}\right) \rightarrow \mu(A)$ then the measure is regular in the corresponding topological measure space.

The inter-relationship between the measurability and Baire categorization of a topological space $X$ is presented in the following theorem where $z \operatorname{er}(A)$ denotes a respective zero-set $A$ within the topological space [11]. 
Theorem 2. A real-valued function $f: X \rightarrow R$ in a topological space $X$ is Baire first category if, and only if, $f($.$) is zer (A)$-measurable.

There is a relationship between the homeomorphism in topological spaces and the multiplicative isomorphism of a semiring structure under the mapping, which is presented in the following theorem [12].

Theorem 3. In a topological space $X$ the function $f(x)=1 / x$ induces the homeomorphism between the topological spaces $(0, \infty],[0, \infty)$ and also induces the multiplicative isomorphism between semirings in $(0, \infty],[0, \infty)$.

The interplay between the convexity of a topological subspace and homeomorphism is illustrated in the following theorem [11].

Theorem 4. Any completely regular topological space $X$ is homeomorphic to a closed subspace $A \subset X$ if $X$ is convex compact, where $A$ is a set of extreme points of the respective topological space.

Note that if the set of extreme points of a topological space $A \subset X$ is Lindelöf, then the Baire first category measurable function $f($.$) exists in A \subset X$, and it can be extended to $X$, which is also Baire first category measurable. Moreover, it is important to note that the Zariski topological space can be established within the Noetherian space, admitting a finite as well as signed Borel measure [14].

\section{Definitions: Hyperconvexity and Measures}

In this paper, $\Lambda \subseteq Z^{+}$denotes an index set, and the topological spaces are Hausdorff as well as first countable. If two topological spaces, $A, B$ are isomorphic, then it is denoted by the algebraic relation $A \cong{ }_{i s o m} B$.

Definition 4 (topological $k$-hyperconvexity). Let $\left(X, \tau_{X}\right)$ be a Hausdorff topological space and $x_{p} \in X$ be a point. An open neighborhood of $x_{p}$ given by $N_{p} \subset X$ is called topologically hyperconvex if $N_{p} \subset \cap \overline{A_{i}}$ where each $\overline{A_{i}}$ is convex in $X$ and $i<+\infty$. A hyperconvex open neighborhood of $\left\{x_{p}\right\} \in \tau_{X}$ in Hausdorff space is denoted by $H_{p} . A H N_{p}$ is called $k-$ hyperconvex if $i \in[1, k]$.

In this paper, we write the hyperconvex subspace to indicate a $k$-hyperconvex subspace for $k>1$. Note that the topological hyperconvexity maintains the countable and finitely boundedness property such that if $i \in I \subset \Lambda$ then $\sup (I)<+\infty$ and $|I|>1$, in general. However, in this case, the finite intersection property excludes the possibilities of attaining $H N_{p}=\phi$ as well as $\left\{x_{p}\right\}=H N_{p}$ where $\left\{x_{p}\right\} \in \tau_{X}$. As a result, the concept of hyperconvex Noetherian class within the topological space $\left(X, \tau_{X}\right)$ can be established, which is defined as follows.

Definition 5 (hyperconvex Noetherian class). Let $x_{p} \in X$ be a point in Hausdorffirst countable topological space $\left(X, \tau_{X}\right)$ with a hyperconvex open neighborhood basis $N B_{p}=\left\{N_{p(k)} \subset X: k \in \Lambda, H N_{p(k)} \cong N_{p(k)}\right\}$ within the space. An open convex collection $S_{p}=\left\{A_{i} \subset X: i \in \Lambda, x_{p} \in A_{i}\right\}$ is called a Noetherian hyperconvex class (NHC) at $\left\{x_{p}\right\} \in \tau_{X}$ if the following properties are satisfied.

$$
\begin{aligned}
& \forall A_{i} \in S_{p}, A_{i}=A_{i}^{o}, \\
& \forall A_{i} \in S_{p}, \exists H N_{p(i)} \exists H N_{p(k)}, \overline{H N_{p(i)}} \subset A_{i}^{o} \subset \overline{\left.H N_{p(k)}\right)} \\
& {[i \leq k] \Rightarrow\left[A_{i} \subseteq A_{k}\right] .}
\end{aligned}
$$

A Noetherian hyperconvex class $S_{p}$ is a relaxed variety such that $\overline{H N_{p(i)}} \neq \overline{A_{i}}$. In other words, $H N_{p(i)}$ need not be locally dense in subspaces in $S_{p}$. The Noetherian hyperconvex class $S_{p}$ is called finite if $i \in I \subset \Lambda$. 
Remark 1. Note that, in general, $S_{p}$ is not a proper neighborhood basis of $\left\{x_{p}\right\} \in \tau_{X}$ although $S_{p}$ is countable. The reason is that if we consider that $X$ is not compact and $I=\Lambda$, then $\exists k \in \Lambda$ such that $\forall i>k, \overline{A_{i}}=\overline{A_{k}}$ in $\left(X, \tau_{X}\right)$ admitting a finite Noetherian class. In an alternative view, it is possible that $\cup\left(A_{i} \in S_{p}\right) \subset X$, where $\left(X, \tau_{X}\right)$ is a compact topological space. In summary, the compactness of a topological space does not influence the nature of finite $S_{p}$.

Note that from now on, if we consider two Hausdorff first-countable topological spaces $\left(X, \tau_{X}\right)$ and $\left(Y, \tau_{Y}\right)$, then the corresponding Noetherian hyperconvex classes at any arbitrary points in two spaces are denoted as $S_{X}$ and $S_{Y}$, respectively. The formation of a neighborhood fiber in a hyperconvex topological subspace at a point $x_{p} \in X$ in the corresponding Noetherian hyperconvex classes $S_{X} \equiv S_{p}$ in $\left(X, \tau_{X}\right)$ is defined as follows.

Definition 6 (neighborhood fiber). Let $\left(X, \tau_{X}\right)$ be a first-countable topological space and $\cup \cup_{i \in \Lambda} A_{i} \subseteq X$ such that $A_{i} \in S_{X}$. A fiber $I \subseteq R, \mu_{p \times I}=\left\{x_{p}\right\} \times I$ at $\left\{x_{p}\right\} \in \tau_{X}$ is a neighborhood fiber if $H N_{p} \subset \underset{\forall i \in \Lambda}{\cap} A_{i}$ is a hyperconvex neighborhood of $x_{p}$.

Remark 2. Note that the condition given by $\exists \overline{H N_{p}} \subset \underset{\forall i \in \Lambda}{\cap} A_{i}$ such that $\exists k \in \Lambda, N_{p(k)} \in N B_{p}$ and $\overline{H N_{p}} \subset H N_{p(k)}$ is maintained in $S_{X} \equiv S_{p}$, where $k<+\infty$. The neighborhood fiber $\mu_{p \times I}$ is a symmetrically compact fiber in $S_{X}$ if $\exists(a \in R) \neq 0, I=[-a, a]$.

If we consider that $\left(X, \tau_{X}\right)$ and $\left(Y, \tau_{Y}\right)$ are two first-countable Hausdorff topological spaces with respective Noetherian hyperconvex class $S_{X}$ and the Noetherian class $S_{Y}$ then it is possible to establish a topological ordering relation $<_{f}$ between the spaces under the function $f:\left(X, \tau_{X}\right) \rightarrow\left(Y, \tau_{Y}\right)$ by considering the closure of subspaces. The definition of topological ordering is defined as follows.

Definition 7 (topological ordering). The Noetherian hyperconvex class $S_{X}$ and the Noetherian class $S_{Y}$ in the respective first-countable Hausdorff topological spaces are topologically ordered if $\forall A_{i} \in S_{X}, \exists B_{i} \in S_{Y}$ such that $f^{-1}\left(\bar{E} \subset B_{i}\right) \subset\left(\bar{F} \subset A_{i}\right)$. The topological ordering is represented as $S_{X}<_{f} S_{Y}$.

It can be observed that the topological ordering relation preserves the concept of continuity of $f:\left(X, \tau_{X}\right) \rightarrow\left(Y, \tau_{Y}\right)$. Later, we will show that $S_{X}<_{f} S_{Y}$ enforces Noetherian hyperconvexity in the codomain of $f:\left(X, \tau_{X}\right) \rightarrow\left(Y, \tau_{Y}\right)$ under homeomorphism. Interestingly, if $\forall A_{i} \in S_{X}$ one can find that $A_{i}=\underset{k \in[1, n]}{\cup} F_{k}, F_{k}=F_{k}^{o}$ such that $n \in \Lambda, n<+\infty$ then $S_{X} \cong \sigma_{s r(X)}$, where $\sigma_{s r(X)}$ is a $\sigma$-semiring in $S_{X}$. As a result, one can consider the corresponding topological space as a topological measure space $\left(X, \sigma_{s r}(X), \mu_{X}\right)$ incorporating a consistent topological measure as defined next.

Definition 8 (NHC measure). A finite measure $\mu_{X}: \sigma_{s r(X)} \rightarrow[0,+\infty)$ is a topological NHC measure in $S_{X}$ if the following conditions are maintained.

$$
\begin{aligned}
& \forall F_{k} \in \sigma_{s r(X)}, \mu_{X}\left(F_{k}\right)<\mu_{X}\left(\overline{F_{k}}\right), \mu_{X}(\{\phi\})=0, \\
& \forall m, n \in \Lambda,\left[A_{m} \subseteq A_{n}\right] \Rightarrow\left[\mu_{X}\left(\overline{A_{m}}\right) \leq \mu_{X}\left(\overline{A_{n}}\right)\right], \\
& {\left[A_{m} \subset A_{n}\right] \Rightarrow\left[\mu_{X}\left(\overline{A_{m} \cup A_{n}}\right)=\mu_{X}\left(\overline{A_{n}}\right)\right] .}
\end{aligned}
$$

Note that the NHC topological measure in a $\sigma$-semiring is measure consistent in local subspaces and also in the global subspaces within the corresponding topological space. The NHC topological measure $\mu_{a e}: \sigma_{s r}(X \cup Y) \rightarrow[0,+\infty)$ is called almost-everywhere in two topological spaces $X, Y$ if $\forall x_{p} \in X, \exists y_{p} \in Y$ it is true that $\exists \overline{N_{x}} \in \sigma_{s r}(X), \exists \overline{N_{y}} \in \sigma_{s r(Y)}$ such that $\mu_{a e}\left(\overline{N_{x}}\right)=\mu_{a e}\left(\overline{N_{y}}\right)$, where $x_{p} \in N_{x}, y_{p} \in N_{y}$ are two respective open neighborhoods and $X \cap Y=\phi$. 
Remark 3. A topological NHC measure in $S_{X}$ generates a non-zero monotone sequence $\Phi\left(S_{X}\right)=\left\langle\mu\left(\overline{A_{i}}\right)\right\rangle_{i=1}^{n}$ determining the compactness as well as convexity of $\left(X, \tau_{X}\right)$. For example, if $\left(X, \tau_{X}\right)$ is a compact and convex topological space, then $n<+\infty, \exists l \in R^{+}$such that $\cup \overline{A_{i}}=X$ and $\Phi\left(S_{X}\right) \rightarrow l>0$. As a result, $\Phi\left(S_{X}\right)$ is bounded and strongly convergent. Otherwise, the sequence $\Phi\left(S_{X}\right)$ is divergent in nature, where $n \rightarrow+\infty$.

\section{Main Results}

The analytical results are presented in two parts as follows. First, we illustrate the topological and measure theoretic properties of sigma-semiring measures in NHC in Section 4.1. The topological separability of sigma-semiring structures in a NHC and the properties of measures are presented in Section 4.2.

\subsection{Properties of Topological NHC Measures}

There is a relationship between the $k$-hyperconvex topological subspaces and the firstcountable property of a Hausdorff topological space. This interrelationship is presented in the following theorem.

Theorem 5. In a topological space $\left(X, \tau_{X}\right)$ if $N B_{p}$ is a finite hyperconvex neighborhood system at $x_{p} \in X$ then it is a Noetherian hyperconvex class if $\left(X, \tau_{X}\right)$ is a first-countable non-compact topological space.

Proof. Let $\left(X, \tau_{X}\right)$ be a first-countable topological space, where $x_{p} \in X$ is an arbitrary point. A local hyperconvex neighborhood system at $x_{p} \in X$ is given by $N B_{p}=\left\{N_{p(k)} \subset X: k \in \Lambda, H N_{p(k)} \cong N_{p(k)}\right\}$ such that one can find a bijection $f: Z^{+} \rightarrow N B_{p}$. The corresponding Noetherian hyperconvex class is $S_{X}$ at $x_{p} \in X$. If we consider that $k \in[1, n<+\infty]$ then we can find a corresponding $l \in[1, m<n]$ such that $\cup_{l}\left(A_{l} \in S_{X}\right) \subset X$ if, and only if, $\left(X, \tau_{X}\right)$ is non-compact and $X \backslash \cup_{l} \overline{A_{l}} \neq \phi$ is open. Moreover, according to the definition $\forall N_{p(k)} \in N B_{p}, \exists A_{l} \in S_{X}$ such that $\overline{N_{p(k)}} \subset A_{l}$ in $\left(X, \tau_{X}\right)$. Inductively, it can be concluded that $[(a \in \Lambda)<(b \in \Lambda)] \Rightarrow\left[N_{p(a)} \subset N_{p(b)}\right]$ and $\exists l \in[1, m]$ such that $A_{l} \subset \overline{N_{p(b)}}$ in non-compact $\left(X, \tau_{X}\right)$. Hence, the local neighborhood system $N B_{p}$ is a Noetherian hyperconvex class where $m<b \leq n$ and $f($.$) is$ finitely countable.

Remark 4. Note that a first-countable topological space may admit a $k$-finite k-hyperconvex class. It is important to note that a non-convex Hausdorff topological space $\left(X, \tau_{X}\right)$ need not always admit a Noetherian hyperconvex class of $N B_{p}$ for $k \in \Lambda$ at any arbitrary $\left\{x_{p}\right\} \in \tau_{X}$ within the space irrespective of the compactness of $X$. The reason is that if $B_{p(k)} \subset X$ is not a convex neighborhood of $x_{p} \in X$ in the compact non-convex $\left(X, \tau_{X}\right)$ then $\underset{k \in \Lambda}{\cup} \overline{B_{p(k)}}=X$; otherwise if $\left(X, \tau_{X}\right)$ is non-convex as well as non-compact, then $\underset{k \in \Lambda}{\cup} \overline{B_{p(k)}} \subset X$. This results in the following corollary, which is a stronger property.

Corollary 1. A Noetherian $\left\{B_{p(n)} \subseteq X, n \in[1, k]\right\}$ admits hyperconvex $N B_{p}$ in a compact Hausdorff and first-countable $\left(X, \tau_{X}\right)$ if, and only if, $\operatorname{Cov}(X)=\left\{X \subset E_{i}, i \in[1, m]\right\}$ is a countable finite cover of $X$, where each $F_{i} \subset E_{i}$ is a convex subcover of $X$.

The topological ordering relation $S_{X}<_{f} S_{Y}$ between the two spaces maintains the respective NHC structures. However, the relation $<_{f}$ also preserves the hyperconvexity in the NHC in the codomain of continuous $f($.$) . The following theorem presents this property.$

Theorem 6. If $\left(X, \tau_{X}\right)$ and $\left(Y, \tau_{Y}\right)$ are first-countable topological spaces with hyperconvex $E \subset X$ and $E<_{f}(F \subset Y)$, then $F$ is also hyperconvex in $Y$. 
Proof. Let $\left(X, \tau_{X}\right)$ and $\left(Y, \tau_{Y}\right)$ be two first-countable topological spaces such that $X \cap Y=\phi$. Suppose $x_{p} \in X$ is an arbitrary point with the corresponding hyperconvex neighborhood basis $N B_{p}$. If $S_{X}$ is a NHC in $\left(X, \tau_{X}\right)$ such that $\forall A_{i} \in S_{X}, x_{p} \in A_{i}$ then $\exists N_{p(n)}, N_{p(m)} \in N B_{p}$ within the topological space, maintaining the property that $[n<m] \Rightarrow\left[\overline{N_{p(n)}} \subset A_{i} \subset \overline{N_{p(m)}}\right]$ in $\left(X, \tau_{X}\right)$. If we consider a continuous function $f:\left(X, \tau_{X}\right) \rightarrow\left(Y, \tau_{Y}\right)$, then $\forall A_{i} \in S_{X}, \exists B_{i} \in S_{Y}$ such that $\left[f^{-1}\left(B_{i}\right) \subset A_{i}\right] \Rightarrow\left[f^{-1}\left(\overline{B_{i}}\right) \subset \overline{A_{i}}\right]$. However, if $A_{i}<_{f} B_{i}$ topological ordering is preserved by $f: S_{X} \rightarrow S_{Y}$ in the two respective topological spaces, then $\left[\bar{E} \subset B_{i}\right] \Rightarrow\left[f^{-1}(\bar{E}) \subset\left(\bar{F} \subset A_{i}\right)\right]$ where $E=E^{o}$ and $F=F^{o}$. Hence, it can be concluded that $E \subset \bigcap_{i \in[1, k]}\left(N_{q(i)} \subset Y\right)$ where $k<+\infty$ such that $B_{i} \subset \overline{N_{q(k)}}$. As a result, $E \subset Y$ is also hyperconvex under $S_{X}<_{f} S_{Y}$.

Note that the converse of Theorem 6 may not always be satisfied under the antisymmetric topological ordering relation, and additional conditions are required. The following lemma is a natural extension of the topological ordering property.

Lemma 1. The topological ordering $S_{X}<_{f} S_{Y}$ preserves homeomorphism of $f:\left(X, \tau_{X}\right) \rightarrow\left(Y, \tau_{Y}\right)$.

There is an interplay between the isomorphisms of the two topological subspaces, topological ordering between the respective NHCs and the corresponding topological measures of the NHCs. The topological ordering in the two NHCs induces an algebraic order between the topological measures in the corresponding NHCs. This property is presented in the following theorem.

Theorem 7. If $S_{X}<_{f} S_{Y}$ is preserved in topological spaces $X \cong{ }_{i s o m} Y$ then $\left(\mu_{a e} \circ f^{-1}\right)(\bar{F})<\mu_{a e}(\bar{E})$ where $F \subset Y$ and $E \subset X$.

Proof. Let $\left(X, \tau_{X}\right)$ and $\left(Y, \tau_{Y}\right)$ be two first-countable Hausdorff topological spaces with respective NHCs $S_{X}, S_{Y}$. Note that the topological spaces are separated as $X \cap Y=\phi$. Suppose we consider $A_{i} \in S_{X}$ and $B_{i} \in S_{Y}$ preserving $S_{X}<_{f} S_{Y}$, which results in $A_{i}<_{f} B_{i}$. If the topological measure $\mu_{a e}: \sigma_{s r}(X \cup Y) \rightarrow[0,+\infty)$ is an almost-everywhere variety and $X \cong{ }_{i s o m} Y$ then the $\mu_{a e}\left(\overline{A_{i}} \in \sigma_{s r(X)}\right)=\mu_{a e}\left(\overline{B_{i}} \in \sigma_{s r(Y)}\right)$ condition is maintained. However, due to the topological ordering $\left(E=E^{o}\right)<_{f}\left(F=F^{o}\right)$ between $\bar{E} \subset A_{i}$ and $\bar{F} \subset B_{i}$ one can conclude that $\left[f^{-1}(\bar{F}) \subset \bar{E}\right] \Rightarrow\left[\left(\mu_{a e} \circ f^{-1}\right)(\bar{F})<\mu_{a e}(\bar{E})\right]$

The above theorem influences the Baire categorization of topological subspaces as illustrated in the following corollary.

Corollary 2. In $S_{X}<_{f} S_{Y}$ and $S_{X} \cong{ }_{i s o m} S_{Y}$ if $\left(\mu_{a e} \circ f^{-1}\right)(\bar{F})<\mu_{a e}(\bar{E})$ then $E \subset X$ and $F \subset Y$ need not be locally dense in $A_{i}$ and $B_{i}$.

Proof. The proof is relatively straightforward because $\mu_{a e}$ is a measure consistently maintaining algebraic ordering $<$ under topological ordering $<_{f}$ even if $(E \cup \partial E) \subset \overline{A_{i}}$ and $(F \cup \partial F) \subset \overline{B_{i}}$.

There is an interplay between the topological ordering and pushforward measure in the two NHCs. Suppose the function $g:\left(X, \sigma_{s r(X)}, \mu_{X}\right) \rightarrow\left(Y, \sigma_{s r}(Y), \mu_{Y}\right)$ is a uniformly measurable function in two isomorphic topological measure spaces. It is interesting to note that the topological ordering $<_{f}$ does not preserve the pushforward measure in NHC under composition with the measurable function $g:\left(X, \sigma_{s r(X)}, \mu_{X}\right) \rightarrow\left(Y, \sigma_{s r(Y)}, \mu_{Y}\right)$. This property is presented in the following theorem, where $f^{-1}$ is the inverse of the corresponding function under the topological ordering relation. 
Theorem 8. If $g:\left(X, \sigma_{s r(X)}, \mu_{X}\right) \rightarrow\left(Y, \sigma_{s r}(Y), \mu_{Y}\right)$ is uniformly measurable in $X \cong_{i s o m} Y$ then $\left(f^{-1} \circ g\right)$ is not a pushforward measure in $S_{X}<_{f} S_{Y}$.

Proof. Let $\left(X, \sigma_{s r(X)}, \mu_{X}\right),\left(Y, \sigma_{s r(Y)}, \mu_{Y}\right)$ be two measure spaces in respective topological spaces, where the $S_{X}<_{f} S_{Y}$ condition is maintained between two NHCs. Suppose $g:\left(X, \sigma_{s r}(X), \mu_{X}\right) \rightarrow\left(Y, \sigma_{s r}(Y), \mu_{Y}\right)$ is a uniformly measurable function with $X \cong_{i s o m} Y$ such that $\mu_{X}\left(\overline{A_{i}} \in \sigma_{s r(X)}\right)=\mu_{X}\left(g^{-1}\left(\overline{B_{i}} \in \sigma_{s r}(Y)\right)\right)$. However, the topological ordering $S_{X}<_{f} S_{Y}$ induces an inequality in measures under composition $\left(f^{-1} \circ g\right)$ which is given by $\mu_{Y}\left(\overline{B_{i}} \in \sigma_{s r}(Y)\right)>\mu_{X}\left(\left(f^{-1} \circ g\right)\left(\overline{A_{i}} \in \sigma_{s r(X)}\right)\right)$. Hence, the condition of the pushforward measure is not preserved by $\left(f^{-1} \circ g\right)$ under $<_{f}$ between the two NHCs.

Although the pushforward measure is not preserved by $<_{f}$ topological ordering between multiple NHCs, the hyperconvex neighborhood system is finitely measurable in each topological measure space, and the topological ordering induces an order in the corresponding measures. This observation is illustrated in the following lemma.

Lemma 2. In every first-countable $\left(X, \tau_{X}\right)$ the topological measure space $\left(X, \sigma_{s r}(X), \mu_{X}\right)$ admits finite measures of hyperconvex neighborhood basis and the topological ordering $S_{X}<_{f} S_{Y}$ between NHCs induces a corresponding order in the neighborhood measures.

Proof. Let $\left(X, \tau_{X}\right)$ be a first-countable topological space, where $N B_{p}=\left\{N_{p(k)} \subset X: k \in \Lambda, H N_{p(k)} \cong N_{p(k)}\right\}$ is an open hyperconvex neighborhood basis. Clearly, $\overline{N B_{p}}$ is countable under the bijection $h: Z^{+} \rightarrow \overline{N B_{p}}$ where $\overline{N B_{p}}=\left\{\overline{N_{p(k)}}\right\}$. As a result, the measure $\left(\mu_{X} \circ h\right) \in(0,+\infty)$ is finite in the corresponding measure space $\left(X, \sigma_{\operatorname{sr}(X)}, \mu_{X}\right)$ where $0<\mu_{X}\left(N_{p(k)}\right)<\mu_{X}\left(\overline{N_{p(k)}}\right)$ by the definition of topological NHC measure. Moreover, if $\left(Y, \tau_{Y}\right)$ is another first-countable topological space with $N B_{q}$ for some $y_{q} \in Y$ then $\left(\mu_{X} \circ f^{-1}\right)\left(\overline{N_{q(k)}}\right)<\mu_{Y}\left(\overline{N_{q(k)}}\right)$ under $<_{f}$ between the topological measure spaces $\left(X, \sigma_{s r}(X), \mu_{X}\right),\left(Y, \sigma_{s r}(Y), \mu_{Y}\right)$.

\subsection{Topological Separation of Sigma-Semiring and Measurability}

It is noted earlier in this paper that the increasing convex functional $\omega: X \rightarrow R \cup\{+\infty\}$ can be formulated in a linear function space $X$, where $\omega$ is convex. However, the measure of the convex bounded measurable functions in a linear function space is finitely additive with the assumption that the sequential semicontinuity of Borel measurable functions is preserved. Note that the convex functional measure can be extended to be infinite. The relationship between the measures and the hyperconvex topological space presented in this paper consider finite measures under the topological decomposition and separation of measure spaces while at least preserving subadditivity. The Hausdorff topological measure space admitting a NHC is considered to be continuous and simply connected in nature.

Let $A_{k}, A_{k-1} \in S_{X}$ be the $k$-hyperconvex and $(k-1)$ - hyperconvex subspaces, respectively, in a NHC in $\left(X, \tau_{X}\right)$. Suppose we consider $E_{((k-1), n)} \subset X$ such that $E_{((k-1), n)}=\left(A_{k} \cup A_{k-1}\right) \backslash \overline{A_{k}}$ where $\overline{A_{k}}=A_{k-1}$ and $n \in \Lambda$. If we take the collection $E_{((k-1), n)}=\bigcup_{i \in[1, n]} D_{((k-1), i)}$ such that $D_{((k-1), i)}=D_{((k-1), i)}^{o}$, then a topological separation of the corresponding $\sigma_{X}-$ semiring is given by the following equation.

$$
\begin{aligned}
& m, n, u \in \Lambda, i \leq u, j \leq u, \\
& \forall E_{((k-m), u),}[i \neq j] \Rightarrow\left[D_{((k-m), i)} \cap D_{((k-m), j)}=\phi\right], \\
& \Omega\left(\sigma_{X}\right)=\left\{A_{k}\right\} \cup\left\{D_{((k-m), u)}\right\}, \\
& \cup E_{((k-m), u)} \subset S_{X} .
\end{aligned}
$$

This immediately leads to the following lemma. 
Lemma 3. $A(k-i)$-hyperconvex subspace is locally dense in the respective component in $\Omega\left(\sigma_{X}\right)$ if, and only if, $i=0$.

The proof of the lemma is directly derivable from the structure of the topologically separated $\sigma_{X}-$ semiring. However, it further results in the following theorem.

Theorem 9. A topologically separated $\Omega\left(\sigma_{X}\right)$ is functionally separable by $\left\{g_{v}: \Omega\left(\sigma_{X}\right) \rightarrow R, v \in \Lambda\right\}$ such that $\bigcap_{v} g_{v}=\phi$ and every $g_{v}($.$) is inner-measurable.$

Proof. Let a topologically separated $\Omega\left(\sigma_{X}\right)$ be in $\left(X, \tau_{X}\right)$ and a set of real-valued functions be given by $\left\{g_{v}: \Omega\left(\sigma_{X}\right) \rightarrow R, v \in \Lambda\right\}$ such that $\bigcap_{v} g_{v}=\phi$. Suppose that the functions in the set maintain the property of local continuity in the topological space by open mapping as $\forall v \in \Lambda, g_{v}\left(W_{v}\right) \subset N_{v}$ such that $N_{v}=N_{v}^{o}$ and $\forall B \subset N_{v}, g_{v}^{-1}(B) \subset W_{v}$ with $B=B^{o}$. As a result, it can be concluded that $[v \neq(u \in \Lambda)] \Rightarrow\left[N_{v} \cap\left(N_{u} \subset R\right)=\phi\right]$. Moreover, as $N_{v}=N_{v}^{o}$ and $W_{v}=W_{v}^{o}$, every $g_{v}($.$) is pushforward inner-measurable due to$ $\left(\mu_{X} \circ g_{v}^{-1}\right)\left(N_{v}\right)<\mu_{X}\left(\overline{W_{v}}\right)$.

Example 1. Let us consider a topological space in $1 D$ such that $A_{k}=(-a, a), A_{k-i}=(-(a+\delta(i)),(a+\delta(i)))$ where $\delta(i)>0, i \in \Lambda$. In this case, the topological separation of the $\sigma_{X}-$ semiring is given by

$$
\begin{aligned}
& i \in \Lambda, n \in Z^{+} \cup\{0\} \\
& \Omega\left(\sigma_{X}\right)=\{(-a, a)\} \cup \\
& \{(-(a+(n+1) . \delta(i)),-(a+n . \delta(i))),((a+n . \delta(i)),(a+(n+1) . \delta(i)))\} .
\end{aligned}
$$

As a result, the topological separation $\Omega\left(\sigma_{X}\right)$ is also separated by $g_{v}: \Omega\left(\sigma_{X}\right) \rightarrow R$ if, and only if, the open neighborhoods under locally continuous mappings are disjoint as $[i \neq j] \Rightarrow\left[\left(N_{i} \subset R\right) \cap\left(N_{j} \subset R\right)=\phi\right]$ where the $\forall\left(B=B^{o}\right) \subset N_{i}, g_{i}^{-1}(B) \subset W_{i}$ condition is preserved. Moreover, every topological separation in $\Omega\left(\sigma_{X}\right)$ is inner-measurable because $\left(\mu_{R} \circ g_{i}\right)\left(W_{i}\right)<\mu_{R}\left(\overline{N_{i}}\right)$ where $\mu_{R}$ is a finite positive measure in reals.

\section{Conclusions}

In a Hausdorff first-countable topological space, the Noetherian hyperconvex class is a generalization of a neighborhood basis without preserving the open property of the singleton element under the intersection of corresponding neighborhoods of that element. The $k$-finite convex intersection generates a $k$-hyperconvex topological subspace admitting a sigma-semiring, which is finitely measurable. The irreflexive, anti-symmetric and transitive topological ordering between two Noetherian $k$-hyperconvex classes retains the homeomorphism of respective topological spaces and induces the ordering in measures in corresponding sigma-semirings. The measure sequence in a Noetherian $k$-hyperconvex class helps in determining compactness of the topological subspace. The measures under the topological ordering do not always preserve the pushforward property, and the sigmasemirings are topologically separable by a set of inner-measurable functions. The concepts presented in this paper may find applications in the topological analysis of dynamics.

Funding: This research is funded by Gyeongsang National University, Jinju, Korea.

Institutional Review Board Statement: Not applicable.

Informed Consent Statement: Not applicable.

Data Availability Statement: Not applicable.

Acknowledgments: The author would like to thank the anonymous reviewers and editors for their valuable comments and suggestions during the peer-review process.

Conflicts of Interest: The author declares no conflict of interest. 


\section{References}

1. Ash, R. The interplay between measure theory and topology. Meas. Integr. Funct. Anal. 1972, 4, 168-200.

2. Spurny, J. Borel sets and functions in topological spaces. Acta Math. Hung. 2010, 129, 47-69. [CrossRef]

3. Hansell, R.W. On the nonseparable theory of Borel and Souslin sets. Bull. Am. Math. Soc. 1972, 78, 236-241. [CrossRef]

4. Stone, A.H. Non-separable Borel sets II. Gen. Topol. Appl. 1972, 2, 249-270. [CrossRef]

5. Mashford, J. A spectral calculus for Lorentz invariant measures on Minkowski space. Symmetry 2020, 12, 1696. [CrossRef]

6. Kirk, R.B.; Crenshaw, J.A. A generalized topological measure theory. Trans. Am. Math. Soc. 1975, 207, 189-217. [CrossRef]

7. Wheeler, R.F. Topological measure theory for completely regular spaces and their projective covers. Pac. J. Math. 1979, 82, 565-584. [CrossRef]

8. Aldaz, J.M.; Render, H. Borel measure extensions of measures defined on sub- $\sigma$-algebras. Adv. Math. 2000, 150, 233-263. [CrossRef]

9. Butler, S.V. Weak Convergence in Topological Measures. J. Theor. Probab. 2021. [CrossRef]

10. Drewnowski, L.; Labuda, I. The Orlicz-Pettis theorem for topological Reisz spaces. Proc. Am. Math. Soc. 1998, 126, 823-825. [CrossRef]

11. Kalenda, O.F.K.; Spurny, J. Extending Baire-one functions on topological spaces. Topol. Its Appl. 2005, 149, 195-216. [CrossRef]

12. Vechtomov, E.M.; Shalaginova, N.V. Semiring of continuous $(0, \infty]$-valued functions. J. Math. Sci. 2018, 233, 28-41. [CrossRef]

13. Cheridito, P.; Kupper, M.; Tangpi, L. Representation of increasing convex functionals with countably additive measures. Studia Math. 2021, 260, 121-140. [CrossRef]

14. Gignac, W. Measures and dynamics on Noetherian spaces. J. Geom. Anal. 2014, 24, 1770-1793. [CrossRef] 